\title{
A TOXINA URÊMICA ÁCIDO GUANIDINICOACÉTICO INIBE O METABOLISMO OXIDATIVO DOS NEUTRÓFILOS DE CÃES
}

\section{UREMIC TOXIN GUANIDINE ACETIC ACID INHIBITS THE OXIDATIVE METABOLISM OF NEUTROPHILS IN DOGS}

\author{
Priscila Preve Pereira ${ }^{1 *}$ \\ Anelise Maria Bosco \\ Breno Fernando Martins de Almeida² \\ Luis Gustavo Narciso² \\ Paulo César Ciarlini \\ ${ }^{1}$ Faculdade de Ciências Agrárias e Veterinárias (FCAV), UNESP, Jaboticabal,SP, Brasil \\ ${ }^{2}$ Faculdade de Medicina Veterinária de Araçatuba (FMVA), UNESP, Araçatuba, SP, Brasil \\ *Autora para correspondência - priscilappvet@gmail.com
}

\begin{abstract}
Resumo
Dentre as toxinas urêmicas que comprovadamente afetam a função neutrofílica na doença renal crônica (DRC) em humanos, destacam-se os compostos guanidínicos. A fim de melhor entender os mecanismos que afetam a imunidade de pacientes urêmicos, no presente estudo foi investigada in vitro a hipótese de que o composto guanidínico ácido guanidinicoacético (AGA) contribui para inibição do metabolismo oxidativo, aumentando a apoptose dos neutrófilos de cães saudáveis. Para tal, neutrófilos isolados de dez cães saudáveis foram incubados em meio de cultura RPMI 1640 puro (controle) e enriquecido com $5 \mathrm{mg} / \mathrm{L}$ de AGA. Utilizando-se citometria de fluxo capilar para a avaliação do metabolismo oxidativo, quantificou-se a produção de superóxido dos neutrófilos empregando-se a sonda hidroetidina, com e sem a presença do estímulo com acetato miristato de forbol (PMA). O índice apoptótico foi quantificado utilizando-se o sistema Anexina V-PE, com e sem o efeito indutor da camptotecina. Os neutrófilos isolados e incubados em meio enriquecido com AGA, quando ativados com PMA, produziram uma menor quantidade de superóxido $(p<0,001)$, porém tal inibição do metabolismo oxidativo ocorreu sem alterar significativamente a viabilidade e a taxa de apoptose. Assim, os resultados evidenciam que os compostos guanidínicos podem contribuir para imunossupressão de cães com DRC.
\end{abstract}

Palavras-chave: explosão respiratória; insuficiência renal; superóxido; uremia.

\begin{abstract}
Among the uremic toxins proven to affect the neutrophil function in humans with chronic kidney disease (CKD), guanidine compounds stand out. To achieve a clearer understanding of the mechanisms that affect the immunity of uremic patients, the hypothesis that guanidine acetic acid (GAA) contributes to the inhibition of oxidative metabolism and an increase in neutrophil apoptosis in healthy dogs was investigated in vitro. To this end, neutrophils isolated from ten healthy dogs were incubated in pure RPMI 1640 (control) and enriched with $5 \mathrm{mg} / \mathrm{L}$ of GAA. Capillary flow cytometry was used to quantify superoxide production in neutrophils with the probe (hydroethidine), in the presence and absence of phorbol-12-myristate-13-acetate (PMA), in order to assess oxidative metabolism. Apoptotic indices were quantified using the Annexin V-PE system, with and without the inductive effect of camptothecin. Neutrophils isolated and incubated in a GAA-enriched medium produced smaller amounts of superoxide $(\mathrm{p}<0.001)$ when activated with PMA, however, this inhibition of oxidative metabolism occurred without significantly altering their viability or rate of apoptosis. Thus,
\end{abstract}


the results show guanidine compounds contribute to immunosuppression in dogs with CKD. Keywords: kidney failure; respiratory burst; superoxide; uremia.

Recebido em: 05 fevereiro 2014

Aceito em: 06 agosto 2015

\section{Introdução}

A formação de compostos oxidantes é fisiologicamente importante como parte do mecanismo de defesa contra micro-organismos invasores. É o caso do ânion superóxido produzido pelos neutrófilos para combater agentes infecciosos e realizar a destruição desses micro-organismos após a fagocitose. Entretanto, quando os compostos oxidantes são gerados de forma excessiva e os mecanismos de defesa antioxidantes são insuficientes, desenvolve-se o estresse oxidativo ${ }^{(1)}$.

Recentemente, foi demonstrado que cães com doença renal crônica (DRC) apresentam estresse oxidativo associado ao aumento ${ }^{(2)}$ ou diminuição ${ }^{(3,4)}$ da produção neutrofílica de superóxido. Tem sido postulado que as toxinas urêmicas promovem inicialmente uma ativação do metabolismo oxidativo e que o excesso de espécies reativas de oxigênio geradas, numa segunda etapa, promove o aumento da apoptose e consequente diminuição da produção de superóxido dos neutrófilos ${ }^{(2,5)}$.

A inter-relação entre as toxinas urêmicas e o estresse oxidativo é um ponto importante na nefrologia ${ }^{(6)}$, bem como a compreensão dos mecanismos imunossupressores que afetam os neutrófilos de pacientes portadores de DRC, predispondo-os a infecções ${ }^{(7,8)}$. Devido ao aumento da síntese dos compostos guanidínicos e ao déficit de excreção renal, esses compostos derivados do metabolismo proteico se destacam entre as demais toxinas urêmicas por atingirem altas concentrações no sangue de pacientes humanos portadores de DRC ${ }^{(9-11)}$. Embora sejam filtráveis, a concentração de compostos guanidínicos ainda permanece elevada em pacientes submetidos à hemodiálise ou diálise peritoneal ${ }^{(12)}$.

Os estudos avaliando o efeito dos compostos guanidínicos na função neutrofílica são escassos e contraditórios. Já se sabe que, in vitro, os compostos guanidínicos (ácido guanidinosuccínico; ácido guanidinopropiônico; ácido guanidinobutírico) comprometem o fluxo de energia da célula prejudicando a produção de superóxido neutrofílica. Curiosamente, nesse mesmo estudo, foi observado que o ácido guanidinicoacético (AGA) foi o único composto guanidínico que não inibiu o metabolismo oxidativo dos neutrófilos humanos ${ }^{(13)}$. Divergindo de tais resultados, já foi demonstrado que o AGA suprime a produção de superóxido em leucócitos humanos ativados ${ }^{(14)}$. Embora a DRC seja comum em cães, os estudos sobre o efeito das toxinas urêmicas na função neutrofílica desta espécie são escassos. Até o momento, não se sabe quais toxinas urêmicas afetam a função dos neutrófilos de cães, nem quais mecanismos estão envolvidos na imunossupressão.

Objetivando ampliar o conhecimento sobre os mecanismos envolvidos na disfunção dos neutrófilos de cães com DRC, foi investigada in vitro a hipótese de que o composto guanidínico AGA isoladamente contribui para a inibição do metabolismo oxidativo dos neutrófilos por meio do aumento da taxa de apoptose dos neutrófilos caninos.

\section{Material e Métodos}

A presente investigação foi realizada de acordo com os princípios éticos em uso de animais do Comitê de Ética em Experimentação Animal, da Universidade Estadual Paulista (Protocolo FOA00762/2012).

Como fonte doadora de neutrófilos, foram selecionados intencionalmente dez cães adultos hígidos de diferentes raças e sexo, sem qualquer alteração nos exames clínico geral e laboratoriais (hemograma completo, urinálise tipo I e bioquímica plasmática). Animais tratados nos últimos 15 dias com qualquer tipo de droga não foram incluídos no estudo.

Após jejum alimentar de 8 a 12 horas, 10 mililitros de sangue total foram colhidos de cada animal por punção da veia jugular, dos quais $9 \mathrm{~mL}$ foram acondicionados em tubos heparinizados estéreis para 
realização do isolamento dos neutrófilos e obtenção de plasma para análises bioquímicas. O restante do sangue foi acondicionado em tubo com $\mathrm{K}_{2}$ EDTA para realização do hemograma.

As análises bioquímicas plasmáticas foram realizadas em espectrofotômetro automatizado (BS 200, Shenzhen Mindray Bio-Medical Eletronics Co., Nanshan, China), previamente calibrado com calibrador e soros controles níveis I e II, e utilizando reagentes comerciais (Biosystems, Barcelona, Espain). Foi determinado o teor de albumina (método verde de bromocresol); aspartato aminotransferase (AST) e alanina aminotransferase (ALT) (ambas pelo método cinético UV segundo IFCC); colesterol (método enzimático oxidase/peroxidase); creatinina (método cinético picrato alcalino); glicose (método enzimático oxidase/peroxidase); ureia (método enzimático UV urease/ glutamato desidrogenase); proteína total (método biureto) e proteína urinária (método vermelho de pirogalol). Todas as reações bioquímicas foram processadas a $37{ }^{\circ} \mathrm{C}$ conforme orientações dos fabricantes.

O hemograma foi realizado em contador de células automatizado veterinário (BC-2800Vet, Shenzhen Mindray Bio-Medical Eletronics Co., Nanshan, China) com a contagem diferencial de leucócitos realizada em esfregaço sanguíneo ${ }^{(15)}$.

A urina foi colhida por cistocentese para realização do exame de urina tipo I e determinação da relação proteína/creatinina urinária (UPC). A densidade urinária foi obtida por refratometria e o exame químico foi realizado utilizando tiras reagentes comerciais (Combur10 test ${ }^{\circledR}$, Roche, Mannheim, Germany). O sedimento urinário foi preparado a partir da centrifugação de $5 \mathrm{~mL}$ de urina a $1.500 \mathrm{rpm}$, o sobrenadante foi armazenado para determinação do UPC. Após homogeneização do sedimento, uma gota foi colocada entre lâmina e lamínula para avaliação em microscopia óptica (400X).

Para avaliação do metabolismo oxidativo e da taxa de apoptose dos neutrófilos, o isolamento dos neutrófilos caninos foi realizado conforme protocolo já descrito ${ }^{(3)}$. Resumidamente, quatro mililitros de sangue heparinizado foram pipetados sobre duplo gradiente de separação contendo três mililitros de Histopaque $1119^{\circledR}$ e três mililitros de Histopaque $1077^{\circledR}$ (Sigma-Aldrich Co., St. Louis, USA), respectivamente. Após centrifugação a $2.000 \mathrm{rpm}$ por 30 minutos, a camada de polimorfonucleares foi lavada duas vezes com solução aquosa de cloreto de amônio $(0,14 \mathrm{M})$ para eliminação dos eritrócitos residuais. Em seguida, foi realizada mais uma lavagem com solução salina tamponada de Hanks (Sigma-Aldrich Co., St. Louis, USA) para bloquear a lise celular. As células foram então diluídas em meio de cultura celular RPMI 1640 (Sigma-Aldrich Co., St. Louis, USA) na concentração final de neutrófilos de $1 \times 10^{6} / \mathrm{mL}$. A viabilidade foi estimada pelo método de exclusão do azul de tripan e a pureza foi determinada por citologia após citocentrifugação. Apenas foram incluídos no estudo os isolamentos que obtiveram viabilidade e pureza iguais ou superiores a 95 e $93 \%$, respectivamente.

Um mililitro da suspensão de neutrófilos $\left(1 \times 10^{6} / \mathrm{mL}\right)$ dos 10 cães selecionados foi incubado em meio RPMI 1640 puro (controle) e com adição do AGA (5 mg/L) (Sigma-AldrichCo., St. Louis, USA) por 4 horas a $37{ }^{\circ} \mathrm{C}$ em termociclador microprocessado (Thermomixer, Eppendorf, Mod. Comfort, Hamburg, Germany). Posteriormente, foram feitas as análises do metabolismo oxidativo e apoptose dos neutrófilos.

O metabolismo oxidativo foi avaliado determinando-se a produção de superóxido dos neutrófilos com a sonda hidroetidina (HE) em citometria de fluxo capilar, segundo metodologia previamente descrita $^{(3)}$. Resumidamente, em um volume de $180 \mu \mathrm{L}$ de suspensão de neutrófilos $\left(1 \times 10^{6} / \mathrm{mL}\right)$ foram acrescidos $20 \mu \mathrm{L}$ de solução tamponada de $\operatorname{HE}(0,1 \mathrm{mM})$ na ausência e presença de estímulo de acetato miristato de forbol 0,55 $\mu \mathrm{M}$ (PMA) (Sigma-Aldrich Co., St. Louis, USA). As soluções foram incubadas em termociclador a $37^{\circ} \mathrm{C}$ por 10 minutos; posteriormente, foram mantidas em banho de gelo, sob proteção da luz, até o momento da leitura. Considerando complexidade e tamanho dos neutrófilos, a população de neutrófilos foi selecionada e a média de fluorescência vermelha de brometo de etídio gerada pela oxidação do HE pelo superóxido foi quantificada em um citômetro de fluxo capilar (Guava EasyCyte Mini ${ }^{\circledR}$, Guava Technologies, Industrial Boulevard Hayward, USA) previamente ajustado para o comprimento de onda máximo para emissão (593 nm) e excitação (473 $\mathrm{nm})$ do brometo de etídio.

A viabilidade e as taxas de apoptose foram estimadas por citometria de fluxo capilar utilizando o sistema anexina V-PE (Guava Nexin Kit, Guava Technologies, USA), conforme previamente descrito $^{(3)}$. Resumidamente, $100 \mu \mathrm{L}$ da solução de neutrófilos $\left(1 \times 10^{6} / \mathrm{mL}\right)$ foi incubada na ausência e na presença do indutor de apoptose camptotecina 6 mM (CAM) (Sigma-Aldrich Co., St. Louis, 
USA) por 10 minutos a $37^{\circ} \mathrm{C}$. Posteriormente, foram adicionados $100 \mu \mathrm{L}$ de anexina V-PE seguindo nova incubação por 20 minutos à temperatura ambiente sob proteção da luz. Após a compensação do citômetro para o fluorocromo vermelho para reação positiva de 7-AAD e do fluorocromo amarelo para reação positiva da anexina V-PE, foi possível quantificar as seguintes populações celulares: células viáveis (anexina $\mathrm{V}-\mathrm{e}$ 7-AAD -), apoptose inicial (anexina $\mathrm{V}+\mathrm{e}$ 7-AAD -) e apoptose final (anexina $\mathrm{V}+\mathrm{e}$ 7-AAD +). A apoptose total foi obtida após soma das populações de células em apoptose inicial e final.

A análise foi realizada em programa computacional específico (Guava Express, CytoSoft Data Acquisition and Analysis Software, Personal Cell Analysis, v.4.1, 2006. Guava Technologies, Industrial Boulevard Hayward, USA) após aquisição de 10.000 eventos.

Após análise das variáveis quanto à normalidade (Teste de Shapiro-Wilk), as diferenças entre os tratamentos foram analisadas pelo teste de $\mathrm{T}$ pareado ou pelo teste de Wilcoxon, considerando significante $\mathrm{p}<0,05$. Toda a análise estastísitca foi realizada em programa específico (SAS Institute Inc., release 9.2, Cary: NC, 2008).

\section{Resultados e Discussão}

Os resultados dos exames hematológicos, bioquímicos e urinálise dos cães saudáveis (Tabela 1) corresponderam aos valores de normalidade da espécie ${ }^{(2,15,16)}$, certificando, assim, a higidez dos animais utilizados como doadores de neutrófilos dos ensaios in vitro.

O AGA inibiu in vitro o metabolismo oxidativo dos neutrófilos de cães saudáveis na presença do estímulo com PMA (Figura 1B) sem alterar viabilidade e apoptose dessas células (Tabela 2). A produção espontânea basal de superóxido não foi afetada pela presença do AGA (Figura 1A).

Tabela 1: Valores de hemograma, bioquímica plasmática e urinálise (média e desvio-padrão) dos cães saudáveis utilizados como doadores de neutrófilos

\begin{tabular}{lcc}
\hline \multicolumn{1}{c}{ Parâmetro } & Animais saudáveis & Referência \\
\hline & Hemograma & \\
Volume globular (\%) & $47,7 \pm 3,3$ & $(37-55)$ \\
Hemácias $\left(10^{12} / \mathrm{L}\right)$ & $6,5 \pm 0,5$ & $(5,5-8,5)$ \\
Hemoglobina $(\mathrm{g} / \mathrm{dL})$ & $15,9 \pm 1,0$ & $(12-18)$ \\
VCM $(\mathrm{fL})^{*}$ & $73,2 \pm 2,9$ & $(60-77)$ \\
CHCM $(\%)^{* *}$ & $33,3 \pm 0,2$ & $(32-36)$ \\
Leucócitos totais $\left(10^{9} / \mathrm{L}\right)$ & $9,9 \pm 2,6$ & $(6,0-17,0)$ \\
& Bioquímica plasmática & \\
Albumina (g/L) & $31,8 \pm 1,6$ & $(26-33)$ \\
ALT (UI/L) & $44,29 \pm 13,2$ & $(21-102)$ \\
AST (UI/L) & $26,1 \pm 8,1$ & $(23-66)$ \\
Colesterol (mg/dL) & $178,3 \pm 51,4$ & $(135-270)$ \\
Creatinina (mg/dL) & $1,07 \pm 0,16$ & $(0,5-1,5)$ \\
Glicose (mg/dL) & $94,9 \pm 10,7$ & $(68-118)$ \\
Proteína total (g/L) & $65,8 \pm 6,3$ & $(54-71)$ \\
Ureia (mg/dL) & $32,6 \pm 8,1$ & $(10-50)$ \\
& Urinálise & \\
Densidade urinária & $1,041 \pm 0,003$ & $(1,030-1,045)$ \\
UPC ${ }^{* * *}$ & $0,2 \pm 0,07$ & $(0-0,3)$ \\
Sedimento urinário & Nada digno de nota & - \\
\hline${ }^{*}$ VCM - Volume corpuscular médio; & & \\
${ }^{* *}$ CHCM - Concentração hemoglobínica corpuscular média; & \\
$* * *$ UPC - Relação proteína/creatinina urinária. & & \\
& &
\end{tabular}


Tabela 2: Viabilidade, apoptose inicial, apoptose final e apoptose total (média e desvio-padrão) de neutrófilos de cães saudáveis incubados em meio RPMI 1640 puro (controle) ou com ácido guanidinicoacético (AGA) na ausência (NE) ou presença de indução com camptotecina (CAM)

\begin{tabular}{lccc}
\hline Parâmetro & Controle & AGA & p-valor \\
\hline Viabilidade NE (\%) & $98,28 \pm 0,61$ & $98,19 \pm 0,49$ & 0,6224 \\
Viabilidade CAM (\%) & $59,35 \pm 7,21$ & $59,42 \pm 7,68$ & 0,7999 \\
Apoptose inicial NE (\%) & $0,24 \pm 0,24$ & $0,19 \pm 0,12$ & 0,3035 \\
Apoptose inicial CAM (\%) & $20,18 \pm 3,40$ & $20,31 \pm 4,23$ & 0,8783 \\
Apoptose final NE (\%) & $0,33 \pm 0,20$ & $0,37 \pm 0,14$ & 0,4354 \\
Apoptose final CAM (\%) & $20,05 \pm 5,02$ & $19,89 \pm 5,58$ & 0,8750 \\
Apoptose total NE (\%) & $0,65 \pm 0,41$ & $0,48 \pm 0,30$ & 0,9646 \\
Apoptose total CAM (\%) & $40,24 \pm 7,07$ & $34,84 \pm 15,84$ & 0,9833 \\
\hline
\end{tabular}
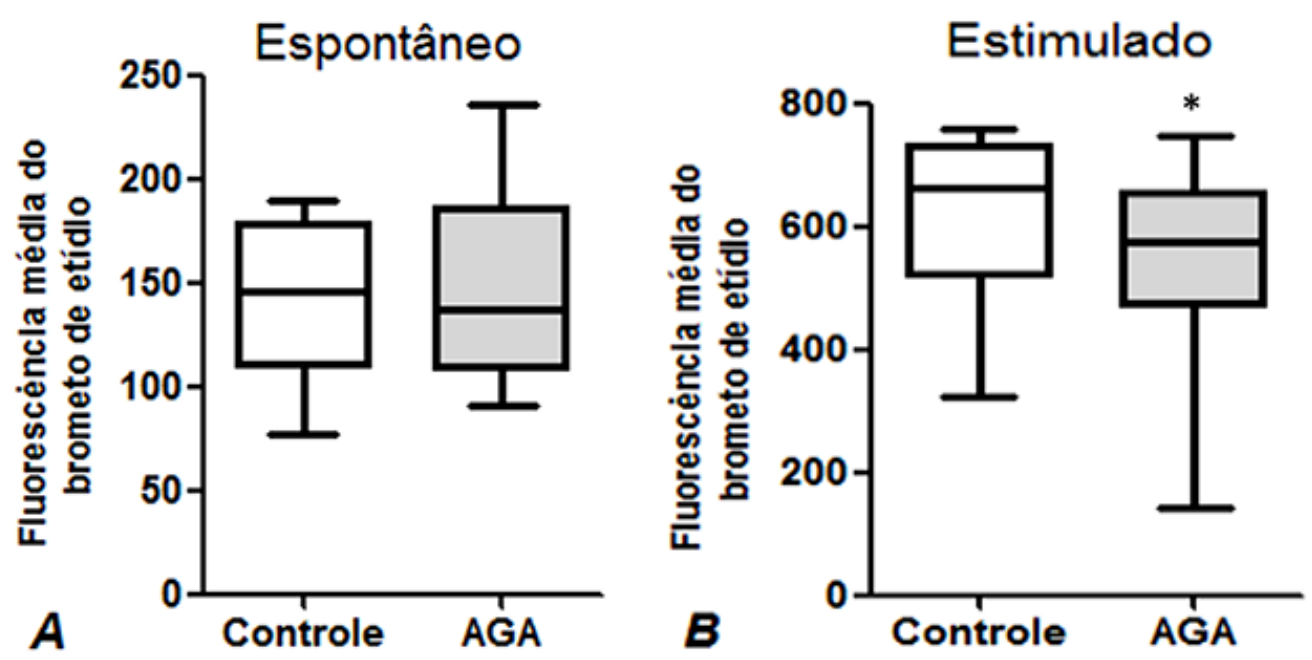

Figura 1: Efeito inibitório do ácido guanidinicoacético (AGA) sobre o metabolismo oxidativo de neutrófilos de cães saudáveis na presença de estímulo. Produção de superóxido estimada pela fluorescência média do brometo de etídio em citometria de fluxo capilar com a sonda hidroetidina em neutrófilos incubados em meio RPMI 1640 puro (Controle) e com AGA (5 mg/L) na ausência (Espontâneo - Figura A) ou presença do estímulo com acetato miristato de forbol (PMA) (Estimulado - Figura B). * p=0,0352.

Em estudos anteriores, o AGA e a metilguanidina, diferentemente dos demais compostos guanidínicos avaliados, não inibiram significativamente o metabolismo oxidativo dos neutrófilos humanos. Ainda segundo os mesmos autores, o efeito inibitório dos demais compostos guanidínicos estava associado à diminuição do aporte de energia necessário para "explosão respiratória" dos neutrófilos. O fato de tal inibição não ocorrer sob o estímulo do PMA pode ser devido ao mecanismo de ativação diferente do zymosan e formil peptídeo met-leu-phe (fLMP), que promove o aumento da produção de superóxido via ativação da proteína quinase $\mathrm{C}$, independentemente do aporte de $\operatorname{ATP}^{(13)}$. Entretanto, tal hipótese não se sustenta diante do efeito inibidor do AGA sobre o metabolismo oxidativo de neutrófilos ativados com PMA observado no presente estudo e também em células mononucleares ${ }^{(14)}$.

A presença de resultados divergentes sobre o efeito de diferentes toxinas urêmicas são comuns, de forma que pesquisadores na área têm procurado estabelecer protocolos para estudos in vitro ${ }^{(11)}$. A comparação dos resultados do presente estudo é prejudicada, uma vez que não existem estudos com cães, apenas com neutrófilos de pacientes humanos saudáveis ${ }^{(13)}$ e linhagem de monócitos humanos ${ }^{(14)}$. Ainda deve ser considerado que há outras diferenças entre os estudos quanto ao delineamento experimental (concentrações e tempo de incubação com as toxinas) e quanto à metodologia utilizada para a ativação e quantificação da produção de superóxido dos neutrófilos. Dessa forma, mais estudos 
são necessários para esclarecer a razão para tais divergências e o mecanismo pelo qual o AGA afeta o metabolismo oxidativo dos neutrófilos de cães.

Identificar os fatores que influenciam a função e a apoptose dos neutrófilos é o primeiro passo para entender a causa da imunossupressão que ocorre na uremia ${ }^{(17)}$. Já se sabe que compostos presentes no plasma de cães urêmicos diminuem in vitro a produção de superóxido de neutrófilos de cães ${ }^{(18)} \mathrm{e}$ que cães com DRC possuem diminuição da produção neutrofílica de superóxido à medida que a apoptose dos neutrófilos aumenta ${ }^{(3)}$. Com base nestes estudos, foi formulada a hipótese inicial de que o efeito inibitório dos compostos guanidínicos sobre o metabolismo oxidativo estaria associado ao aumento da apoptose dos neutrófilos. Porém o AGA promoveu a diminuição da produção de superóxido (Figura 1B) sem acelerar a apoptose dos neutrófilos (Tabela 2). Esses resultados sugerem que o AGA isoladamente não contribui para o maior estresse oxidativo ou aumento da apoptose dos neutrófilos já descritos em cães com $\mathrm{DRC}^{(2,3)}$. O efeito inibitório do AGA sobre a produção neutrofílica de superóxido obtida in vitro dificilmente poderá ser confirmado in vivo nos cães com DRC, pois durante a síndrome urêmica dezenas de outras toxinas agem provavelmente de modo distinto sobre a função dos neutrófilos ${ }^{(17)}$.

$\mathrm{O}$ presente estudo registra uma das primeiras evidências de que os compostos guanidínicos, à semelhança do que ocorre em humanos, são potencialmente capazes de afetar a função de neutrófilos em cães. A fim de se tentar preservar a resposta imune de cães com DRC, torna-se necessário o melhor entendimento de como o AGA e outras toxinas urêmicas afetam a função dos neutrófilos. Dessa forma, poderão ser estabelecidas novas estratégias de tratamento, incluindo o desenvolvimento de membranas de hemodiálise com maior capacidade de filtrar compostos guanidínicos, ou mesmo de drogas capazes de inibir a produção dessas toxinas.

\section{Conclusão}

O composto guanidínico AGA é capaz de promover a inibição do metabolismo oxidativo de neutrófilos in vitro sem afetar a viabilidade dessas células.

\section{Agradecimentos}

À FAPESP pelo financiamento do projeto (Proc. 2011/18746-3) e Laine Margareth Gabas pelo auxílio na pesquisa.

\section{Referências}

1. Kao MPC, Ang DSC, Pall A, Struthers AD. Oxidative stress in renal dysfunction: mechanisms, clinical sequelae and therapeutic options. Journal of Human Hypertension. 2010;24(1):1-8.

2. Almeida BFM, Narciso LG, Melo LM, Pereira PP, Bosco AM, Lima VM, Ciarlini, PC. Leishmaniasis causes oxidative stress and alteration of oxidative metabolism and viability of neutrophils in dogs. The Veterinary Journal. 2013;198(3):599-605.

3. Silva ACRA, Almeida BFM, Soeiro CS, Ferreira WL, Lima VMF, Ciarlini PC, Oxidative stress, superoxide production, and apoptosis of neutrophils in dogs with chronic kidney disease. Canadian Journal of Veterinary Research. 2013;77(2):136-141.

4. Almeida, BFM, Narciso LG, Bosco AM, Pereira PP, Braga ET, Avanço SV, Marcondes M, Ciarlini PC. Neutrophil dysfunction varies with the stage of canine visceral leishmaniosis. Veterinary Parasitology. 2013;196(1-2):6-12.

5. Cendoroglo M, Jaber, BL, Balakrishnan, VS, Perianayagam M, King AJ, Pereira BJG. Neutrophil apoptosis and dysfunction in uremia. Journal of the American Society of Nephrology. 1999;10(1):93-100.

6. Rutkowski P, Malgorzewicz S, Slominska E, Renke M, Szydlowska WL, Swierczynski J, Rutkowski B. Interrelationship between uremic toxicity and oxidative stress. Journal of Renal Nutrition. 2006;16(3):190193.

7. Hörl WH. Neutrophil function and infections in uremia. American Journal of Kidney Diseases. 1999;33(2):4548. 
8. Cohen G, Rudnicki M, Höorl WH. Uremic toxins modulate the spontaneous apoptotic cell death and essential functions of neutrophils. Kidney International. 2001;78(59):548-552.

9. De Deyn PP, Marescau B, Cuykens JJ, Gorp LV, Lowenthal A, De Potter WP Guanidino compounds in serum and cerebrospinal fluid of non-dialyzed patients with renal insufficiency. Clinica Chimica Acta. 1987;167(1):81-88.

10. Tanaka A, Takahashi Y, Mizokuchi M, Shimada N, Koide H. Plasma, urinary, and erythrocyte concentrations of guanidino compounds in patients with chronic renal failure. Renal Failure. 1999;21(5):499-514.

11. Vanholder R, Smet R, Glorieux G, Argilés A, Baurmeister U, Brunet P, Clark W, Cohen G, De Deyn PP, Deppisch R, Descamps-Latscha B, Henle T, Jörres A, Lemke HD, Massy ZA, Passlick-Deetjen J, Rodriguez M, Stegmayr B, Stenvinkel P, Tetta C, Wanner C, Zidek W, and E.U.T.W.G. (EUTox). Review on uremic toxins: classification, concentration, and interindividual variability. Kidney International. 2003;63(5):19341943.

12. De Deyn P, Marescau B, Lornoy W, Becaus I, Van Leuven I, Van Gorp L, Lowenthal A. Serum guanidino compound levels and the influence of a single hemodialysis in uremic patients undergoing maintenance hemodialysis. Nephron. 1987;45(4):291-295.

13. Hirayama A, Dutra AAN, Gordge MP, Neild GH, Hothessall JS. Inhibition of neutrophil superoxide production by uremic concentrations of guanidino compounds. Journal of the American Society of Nephrology. 2000;11(4):684-689.

14. Glorieux G, Dhondt AW, Jacobs P, Langeraert JV, Lameire NH, De Deyn PP, Vanholder RC. In vitro study of the potential role of guanidines in leukocyte functions related to atherogenesis and infection. Kidney International. 2004;65(6): 2184-2192.

15. Rizzi TE, Meinkoth JH, Clinkenbeard KD. Normal Hematology of the Dog. In: Weiss DJ, Wardrop KJ. Schalm's Veterinary Hematology. 6th ed. Philadelphia: Wiley-Blackwell; 2010. p. 799-810. English.

16. Kaneko JJ, Harvey, JW, Bruss ML. Clinical biochemistry of domestic animals. 6th ed. London: Academic Press; 2008. 904p. English.

17. Cohen G, Höll WH. Immune dysfunction in uremia-an update. Toxins. 2012;4(11):962-990.

18. Barbosa TS, Mori CK, Ciarlini PC. Efeito inibidor do soro urêmico sobre o metabolismo oxidativo dos neutrófilos de cães. Arquivo Brasileiro de Medicina Veterinária e Zootecnia. 2010;62(6):1352-1358. 